\title{
¿Cuáles son las expectativas de los profesionales de Educación Superior?
}

\author{
What are the expectations of higher education \\ professionals?
}

\author{
Oldemar Villatoro Moya \\ Universidad Nacional \\ Puntarenas, Costa Rica \\ oldemar.villatoro.moya@una.cr
}

Recibido: 16/05/2018 Aceptado: 30/08/2018

Resumen. La calidad de la educación debe ser de forma permanente en las aulas, en todas las instituciones de enseñanza superior. Por lo tanto, se requiere que los profesionales de la educación sean reflexivos e investigadores, comprometidos con la investigación y la acción social. Deben pensar críticamente sobre los supuestos educativos, los valores y la práctica en un mundo de cambio constante en aras de la mejora continua. La evidencia se debe reflejar en la investigación como parte de la práctica pedagógica. Los profesionales de la educación superior deben poseer características, aptitudes, actitudes y sobre todo actuar con honestidad e integridad, lo cual permitirá demostrar altos estándares de ética y apoyo a los estudiantes y así cumplir con las expectativas. Los formadores son profesionales duales; son especialistas vocacionales y expertos en enseñanza y aprendizaje. Están comprometidos a mantener y desarrollar experiencia en ambos aspectos de su función para garantizar lo mejor para el estudiantado. Los profesionales de la educación deben cumplir con una serie de requerimientos, por ejemplo: ser buenos líderes, identificar problemas, ser creativos, inmersos en el autoaprendizaje y desarrollo personal, comprometidos con la calidad y el saber comunicarse con los demás.

Palabras clave: autoaprendizaje, calidad, conocimiento, habilidades, liderazgo, profesional. 


\begin{abstract}
The quality of education must be permanently protected and ensured within classrooms throughout all institutions of higher education. To guarantee this, education professionals are required to be reflective and research-oriented, committed to continuous learning as well as social action. They must think critically about educational assumptions, values, and practice in a world of constant change for the sake of continuous improvement. The evidence should be based on research as part of the pedagogical practice. Higher education professionals must possess solid characteristics, aptitudes, and attitudes, and, above all, act with honesty and integrity, which will allow demonstrating high standards of ethics and support to students and thus meet educational expectations. The teachers are dual professionals; they are vocational specialists as well as experts in teaching and learning. They are committed to maintaining and developing experience in both aspects of their role to ensure the best for their students. Educational professionals must meet a series of requirements, for example, to be inspiring leaders, problem solvers, creative, immersed in self-learning and personal development, committed to quality, and excellent communicators.
\end{abstract}

Keywords: knowledge, leadership, professional, quality, self-learning, skills.

Un profesional de la educación debe demostrar compromiso en la práctica profesional. Requiere tener valores y atributos, desarrollar su propio juicio sobre lo que funciona y lo que no funciona en la enseñanza. Debe tener conocimiento, habilidades y comprensión profesional. Debe ampliar y desarrollar conocimientos y comprensión profunda y críticamente informada en la teoría y en la práctica.

Es necesario describir los requerimientos que se esperan del profesional de la educación para desarrollar adecuadamente sus funciones, roles, etc., que se derivan del actuar como profesional. Las habilidades profesionales que desarrolle con base en la experiencia le permitirán asegurar los mejores resultados en los estudiantes, por lo tanto, es importante describir cuáles son las habilidades y los conocimientos que deben ser características propias del profesional de la educación.

Los profesionales de la educación deben ser reflexivos e investigadores que piensan críticamente acerca de sus propios supuestos educativos, los valores y la práctica en el contexto de un mundo de cambio contemporáneo y educativo. Muchos se basan en la investigación pertinente como parte de la práctica basada en la evidencia.

Deben actuar con honestidad e integridad para mantener los altos estándares de ética y apoyo a los estudiantes y sus expectativas. Los formadores son profesionales duales; son especialistas vocacionales y expertos en enseñanza y aprendizaje. Están comprometidos con mantener y desarrollar experiencia en ambos aspectos de su función para garantizar lo mejor para los estudiantes. 
URL: http://www.revistas.una.ac.cr/index.php/dialogo/index

CORREO ELECTRÓNICO: universidadendialogo@una.cr

DOI: https://doi.org/10.15359/udre.9-1.5

De acuerdo con Martín (2001), los profesionales de la educación deben cumplir con una serie de requerimientos que se mencionan a continuación:

- Liderazgo

- Identificación de problemas

- Creatividad

- Autoaprendizaje y desarrollo personal

- Calidad

- Comunicación

Estos requerimientos necesitan habilidades y conocimientos. Habilidad se refiere al saber hacer; según la RAE, la habilidad consiste en la "capacidad y disposición para algo" (2017). Por lo tanto, un profesional en la educación debe saber enseñar, debe hacer cosas para enseñar. También, la RAE define conocimiento como la "acción y efecto de conocer" (2017). Estas dos cualidades van de la mano en cuanto a lo que se requiere de un profesional de la educación.

De acuerdo con Mas Torelló (2011), el profesional de la educación tiene un doble perfil (triple, si considerásemos la gestión), donde la formación puede y debe colaborar en

Aumentar el conocimiento correspondiente a su propia área y, del mismo modo, mejorar sus competencias didácticas. Aumentar las competencias innovadoras e investigadoras, especialmente en la vertiente pedagógica, sobre su propia actividad docente (aspecto que lleva asociado la necesidad de una consistente formación pedagógica previa), ya que la formación de su propia área de conocimiento en este ámbito correspondería mayoritariamente (...) a los estudios de doctorado. (p. 204)

El profesional de la educación debe ser competente, debe tener arraigo a "las dos principales funciones profesionales que debe cumplir" (Mas Torelló, 2011, p. 203), estas son: la docencia y la investigación. Son compromisos adquiridos al empezar a ejercer su profesión, además su formación debe ser continua, no puede ni debe quedarse estancado. La mejora continua llevará al profesional a incrementar la calidad del proceso de enseñanza-aprendizaje.

Seguidamente, se describirán los requerimientos primordiales que deben caracterizar al profesional en educación, estos requerimientos nos llevan a identificar cada uno de los conocimientos y las habilidades que se espera que tengan los profesionales en educación. Martín (2001) describe cada uno de los requerimientos antes mencionados: 
- Liderazgo: Se refiere a la capacidad de desarrollar una visión global de la institución y aunar voluntades en torno a un proyecto en común.

- Identificación de problemas: Se refiere a la capacidad de identificar, analizar y relacionar problemas complejos, así como el dominio de las herramientas adecuadas para el tratamiento de los mismos.

- Creatividad: Se refiere al dominio de técnicas de creatividad aplicadas a la innovación.

- Autoaprendizaje y desarrollo personal: Se refiere a la posesión de habilidades y conocimientos para mejorar la eficacia personal, gestionar el desarrollo profesional propio y asesorar a otros.

- Calidad: Se refiere a las habilidades y conocimientos para impulsar y liderar proyectos de calidad, así como para la aplicación de herramientas de calidad.

- Comunicación: Se refiere al nivel de habilidades y conocimientos que permiten diseñar políticas de comunicación para la participación y el consenso, así como hacer presentaciones en público y dirigir reuniones de grupo.

A continuación, se describirán cada uno de los conocimientos y habilidades por cada requerimiento adaptado de Martín (2001).

\section{Liderazgo}

\section{Habilidades}

- Capacidad para dirigir personas: Tiene que saber hacer, de modo que las personas consigan resultados a nivel individual y en grupo, las instituciones educativas obtienen resultados cuando los profesionales en educación saben dirigir a los estudiantes hacia la consecución de los resultados.

- Implicación personal: Saber mantener actitudes y comportamientos que demuestran el compromiso constante del profesional de la educación. Existe un desgaste inevitable en el ejercicio de la docencia, muchas decisiones son difíciles de tomar y con el tiempo se debilitan las actuaciones, sacrificando parcialmente los resultados.

- Capacidad para dirigir reuniones: La mayor parte del conocimiento de las instituciones, así como sus ideas y experiencia, pasan por las reuniones de grupo; siempre que se conduzcan adecuadamente, la utilización de conocimientos e ideas se hace mediante la puesta en común a través de reuniones. 
URL: http://www.revistas.una.ac.cr/index.php/dialogo/index

CORREO ELECTRÓNICO: universidadendialogo@una.cr

DOI: https://doi.org/10.15359/udre.9-1.5

\section{Conocimientos}

- Liderazgo

- Motivación positiva

- Asesoramiento individual (coaching)

- Delegación formal de autoridad y responsabilidad (enpowerment)

\section{Identificación de problemas}

\section{Habilidades}

- Capacidad de análisis: Saber distinguir y separar los datos en una situación compleja, para tener un conocimiento claro de la realidad. El ejercicio profesional requiere capacidad para estudiar situaciones reales.

- Capacidad de relación, visión global del sistema: Debe saber plantear de forma ordenada y cuantificada el conjunto de factores que afectan a una determinada situación. Al análisis de un determinado problema ha de seguir el expresar con claridad las relaciones que existen entre los diversos procesos y subprocesos, lo que resulta esencial para otorgar a cada factor la importancia que tiene.

- Capacidad de síntesis: Habilidad para resumir materias o situaciones complejas. La habilidad de sintetizar un trabajo de análisis y relación posibilita la comunicación, en el trabajo en equipo y el uso del lenguaje entendido por todos.

\section{Conocimientos}

- Resolver diferentes tipos de problemas: problemas individuales, colectivos, internos, externos, organizativos, procesos, productos, servicios, etc.

- Aplicación de procesos y seguimiento de indicadores

- Técnicas de análisis de documentación, presentación, seguimiento y difusión de resultados.

\section{Creatividad}

\section{Habilidades}

- Capacidad de innovación y cambio: Habilidad para modificar una situación introduciendo elementos distintos a los contemplados por el sistema. 
- Desarrollo de pensamiento inductivo: Capacidad de llegar a los principios a partir del conocimiento de datos, mediante un método lógico ascendente. Esta habilidad es necesaria para la aplicación de técnicas que se usan individualmente o en grupo para la búsqueda de soluciones creativas.

\section{Conocimientos}

- Tormenta de ideas

- Análisis de Pareto

- Diseño de escenarios

- Análisis de campos de fuerzas

- Método Delfos

- Diagrama de causa-efecto

- Histogramas

\section{Autoaprendizaje}

\section{Habilidades}

- Capacidad autocrítica: Capacidad para realizar un diagnóstico objetivo de las propias carencias en orden a la consecución de objetivos fijados. El mercado laboral demanda profesionales dispuestos a poner los medios para solucionar sus carencias formativas, que el avance tecnológico y la competitividad convierten en una necesidad permanente. El principal recurso con que cuenta el profesional en educación es el propio conocimiento de sus necesidades.

- Saber buscar información: Capacidad crítica para seleccionar las fuentes y los datos. La selección acertada de los medios de aprendizaje (libros, cursos, información, consultoría, etc.) es un factor crítico elemental para el buen uso del tiempo disponible.

- Orientación práctica: Capacidad para aplicar conceptos, estructuras teóricas y nuevos conocimientos a situaciones reales. El mercado laboral pide aplicaciones prácticas y solución de problemas reales.

\section{Conocimientos}

- Técnicas de autodiagnóstico

- Búsqueda de información en internet, intranet

- Técnicas de estudio individual 


\section{Calidad}

\section{Habilidades}

- Capacidad de relación, visión global: Saber plantear de forma ordenada y cuantificada el conjunto de factores que afectan una determinada situación. La formación en calidad favorece la habilidad de analizar las relaciones que existen entre los diversos elementos que definen la excelencia. Con la adecuada preparación práctica se puede conseguir una considerable habilidad para describir sistemas, lo que resulta esencial para otorgar a cada factor la importancia que tiene.

- Convertir en hábito la mejora continua: Incorporar a la actividad ordinaria el uso de las técnicas que facilitan la aplicación de la gestión de calidad total. La implementación de modelos de calidad en las instituciones exige, además de los conocimientos teóricos, el uso de herramientas comunes que permitan conseguir los objetivos.

\section{Conocimientos}

- Conceptos básicos en gestión total de calidad (TQM)

- Introducción a las normas ISO de aseguramiento de la calidad

- Formación en el modelo de excelencia personal en la educación

- Las herramientas de la calidad

\section{Comunicación}

\section{Habilidades}

- Corrección en la expresión oral y escrita: Saber transmitir el mensaje con precisión. La totalidad práctica de los trabajos que han de desempeñar los docentes exigen comunicar mensajes, con carácter individual o colectivo, de modo público o privado.

- Habilidades de motivación, persuasión y negociación: Capacidad de escucha, asertividad y empatía. En el ámbito laboral es absolutamente necesaria la capacidad de transmitir ideas y soluciones frente a alternativas más o menos coherentes, especialmente en situaciones difíciles.

- Saber buscar información: Capacidad crítica para seleccionar las fuentes y los datos, una comunicación que no esté basada en fundamentos sólidos degenera en retórica, cuyas posibilidades de éxito en el ámbito laboral son nulas. 


\section{Conocimientos}

- En el plano teórico o filosófico

- Habilidades comunicativas

- Ortografía

- Presentación de proyectos

- Técnicas de exposición

- Hablar en público

- Redacción de actas, informes, proyectos

Estos requerimientos son características que deben ser propias del profesional de la educación para el beneficio del educador y de los estudiantes.

Como conclusión, un profesional de la educación que no cumpla con los requerimientos, como poseer habilidades y conocimientos fundamentales, pondrá en cuestionamiento la calidad de la educación, perjudicándose a sí mismo y a los estudiantes. Genera un efecto negativo en el proceso de enseñanzaaprendizaje. Hoy en día se requiere que este proceso sea innovador y de cambio. El profesional de la educación se debe centrar en el logro de sus competencias con un único propósito de alcanzar la calidad y la excelencia educativa.

\section{Referencias bibliográficas}

Martín, E. M. (2001). Gestión de instituciones educativas inteligentes. Madrid: McGraw-Hill.

Mas Torelló, O. (diciembre, 2011). El profesor universitario: sus competencias y formación. Profesorado, 15(3).

Real Academia Española (2017). Diccionario de la lengua española. Recuperado de http://dle.rae 